\title{
Correction to: In vitro regeneration of mulberry plants from seedling explants of Morus indica cv. G4 through direct organogenesis
}

\author{
Tanmoy Sarkar $^{1}{ }^{1} \cdot$ K. N. Ravindra ${ }^{1} \cdot$ S. Gandhi Doss ${ }^{1}$ · P. M. Pratheesh Kumar ${ }^{1} \cdot$ Pankaj Tewary $^{1}$
}

Published online: 31 August 2021

๑ The Author(s), under exclusive licence to Springer-Verlag GmbH Germany, part of Springer Nature 2021

\section{Correction to: Trees}

https://doi.org/10.1007/s00468-021-02186-9

The table 1 has been published with corrections in the original publication. The complete corrected Table 1 is given below. The original article has been corrected. s00468-021-02186-9.

Tanmoy Sarkar

tanmoy.dgr@gmail.com

1 Central Sericultural Research \& Training Institute (CSR\&TI), Mysuru 570008, Karnataka, India 
Table 1 Effects of various concentrations of TDZ on the formation of shoot primordia from cotyledons and hypocotyls during the two phases of pre-culturing

First pre-culturing of explants for 2 days

\begin{tabular}{|c|c|c|c|c|c|c|c|c|}
\hline Treatment & $\mathrm{T} 1$ & & $\mathrm{~T} 2$ & & $\mathrm{~T} 3$ & & $\mathrm{~T} 4$ & \\
\hline Media composition & MS medium & without TDZ & $\begin{array}{l}\text { MS medium } \mathrm{p} \\
\text { TDZ }\end{array}$ & lus $0.1 \mathrm{mg} / \mathrm{L}$ & $\begin{array}{l}\text { MS medium pl } \\
\text { TDZ }\end{array}$ & Is $1.1 \mathrm{mg} / \mathrm{L}$ & $\begin{array}{l}\text { MS medium } \mathrm{p} \\
\text { TDZ }\end{array}$ & us $0.1 \mathrm{mg} / \mathrm{L}$ \\
\hline & Cotyledon & Hypocotyl & Cotyledon & Hypocotyl & Cotyledon & Hypocotyl & Cotyledon & Hypocotyl \\
\hline $\begin{array}{l}\text { Induction of Shoot } \\
\text { primordia }(\%)\end{array}$ & 0.00 & 0.00 & 0.00 & 0.00 & 0.00 & 0.00 & 0.00 & 0.00 \\
\hline Second pre-culturin & of explants fo & 3 days & & & & & & \\
\hline Treatment & $\mathrm{T} 1$ & & $\mathrm{~T} 2$ & & $\mathrm{~T} 3$ & & $\mathrm{~T} 4$ & \\
\hline Media composition & MS medium & without TDZ & $\begin{array}{l}\text { MS medium } \mathrm{p} \\
\text { TDZ }\end{array}$ & us $0.1 \mathrm{mg} / \mathrm{L}$ & $\begin{array}{l}\text { MS medium pl } \\
\text { TDZ }\end{array}$ & is $1.1 \mathrm{mg} / \mathrm{L}$ & $\begin{array}{l}\text { MS medium } \mathrm{p} \\
\text { TDZ }\end{array}$ & us $1.1 \mathrm{mg} / \mathrm{L}$ \\
\hline & Cotyledon & Hypocotyl & Cotyledon & Hypocotyl & Cotyledon & Hypocotyl & Cotyledon & Hypocotyl \\
\hline $\begin{array}{c}\text { Induction of Shoot } \\
\text { primordia }(\%)\end{array}$ & $0.00 \pm 0.00^{\mathrm{e}}$ & $0.00 \pm 0.00^{\mathrm{e}}$ & $53.33 \pm 3.33^{b}$ & $8.88 \pm 1.11^{\mathrm{de}}$ & $57.04 \pm 5.00^{\mathrm{ab}}$ & $15.29 \pm 3.56^{\mathrm{cd}}$ & $65.55 \pm 2.94^{\mathrm{a}}$ & $22.22 \pm 2.22^{\mathrm{c}}$ \\
\hline
\end{tabular}

Data represent mean \pm standard error $(\mathrm{SE})$ of three repeated experiments, each with 25 explants per explant type. The values followed by different letters for each variable are significantly different at $p \leq 0.05$ according to Tukey's multiple comparison test

Publisher's Note Springer Nature remains neutral with regard to jurisdictional claims in published maps and institutional affiliations. 